\title{
Long Term Complete Response of Unresectable Locally Advanced Pancreatic Cancer after CCRT and Gemcitabine Chemotherapy
}

\author{
Jaeyun Yang, Taekyu Lim, Taegyoon Kim, Seungmoon Han, Sanghee Lee, Huiseo Kim, Jiwon Lee, Seongyeong Ahn \\ Department of Internal Medicine, Veterans Health Service Medical Center, Seoul, Korea
}

Locally advanced or metastatic disease accounts for two thirds of total patients with pancreatic cancer. Patients with pancreatic cancer are assessed as resectable, potentially resectable (borderline) or unresectable according to pre-operative examinations. The chances of resectability may be enhanced by using neoadjuvant systemic chemotherapy, radiotherapy or both. This case report presents a locally advanced pancreatic adenocarcinoma that was identified to be unresectable during surgical exploration. After receiving concurrent chemoradiotherapy, the patient was re-evaluated, identified as unresectable and received gemcitabine maintenance chemotherapy. Herein, we report the case of a patient with unresectable locally advanced pancreatic adenocarcinoma who achieved a complete response lasting for more than 32 months after receiving concurrent chmoradiotherapy followed by gemcitabine maintenance chemotherapy.

Korean J Pancreas Biliary Tract 2016;21(4):209-215

Keywords: Pancreatic cancer, Chemoradiotherapy, Gemcitabine, Chemotherapy

\author{
Received Apr. 26, 2016 \\ Revised Jun. 27, 2016 \\ Accepted Jul. 17, 2016
}

Corresponding author : Taekyu Lim

Department of Internal Medicine, Veterans Health Service Medical Center, 53 Jinhwangdo-ro 61gil, Dunchon-dong, Gangdong-gu, Seoul 05368, Korea

Tel. +82-2-2225-1669 Fax. +82-2-2225-4374

E-mail; imegene@naver.com

This is an Open Access article distributed under the terms of the Creative Commons Attribution Non-Commercial License (http:// creativecommons.org/licenses/by-nc/3.0/) which permits unrestricted non-commercial use, distribution, and reproduction in any medium, provided the original work is properly cited.

Copyright @ 2016 by The Korean Journal of Pancreas and Biliary Tract

\section{INTRODUCTION}

Pancreatic cancer is the fourth leading cause of cancer-related death, and accounts for nearly $3 \%$ of new cancer diagnosed, with a worldwide incidence reaching approximately to 217,000 new cases per year. ${ }^{1,2}$ Complete resection is the only potentially curative treatment. ${ }^{3}$ However, only 10 to 20
$\%$ of the patients fulfill the criteria of primarily resectable disease at diagnosis, as they are usually diagnosed at an advanced stage with or without metastasis. ${ }^{4,5}$ Median overall survival reaches only 5-7 months among patients with unresectable disease, whereas patients after complete resection combined with neo- or adjuvant treatment survive about 2 years. ${ }^{6,7}$ Hence, survival rate will greatly increase if resect- 
ability is achieved in unresectable pancreatic cancer.

We would report a case that an unresectable pancreatic cancer patient treated with neoadjuvant concurrent chemoratiotherapy (CCRT) followed by gemcitabine maintenance chemotherapy achieved a long term complete response.

\section{CASE}

A 64 year-old man who had been healthy visited local clinic with symptoms of polydipsia and polyuria in October 2010 and was diagnosed with diabetes mellitus. Thereafter, he had regular checkup in spring 2011 and there were elevated levels of carbohydrate antigen (CA) 19-9 (176.29 U/mL) on laboratory tests. He complained no other symptoms at the time. But, abdominopelvic computed tomography (AP-CT) was performed for further evaluation since potential for pancreatic cancer could not be excluded with taking diabetes mellitus newly diagnosed at the old age and a significant increase in CA 19-9 levels into account. And he was suspected with $2.9 \mathrm{~cm}$ sized pancreatic body cancer on the AP-CT (Fig. 1). The AP-CT showed no cancer invasion in the celiac axis, superior mesenteric artery, common hepatic artery and no distant metastasis. But there was cancer invasion in the superior mesenteric vein (SMV)-portal vein (PV) confluence on the AP-CT.
Cancerous lesion on the pancreatic body was also revealed with invasion in the SMV-PV confluence by magnetic resonance cholangiopancreatography (MRCP) (Fig. 1). Based on these findings, the patient was diagnosed with stage IIA (cT3N0M0) pancreatic cancer according to American Joint Committee on Cancer (AJCC) staging criteria. Surgical exploration was performed to evaluate the chance of resectability in spite of unresectability on imaging study. But this revealed findings that are suggestive of cancer invasion in the SMV-PV confluence and common hepatic artery. The patient was therefore assumed to have an unresectable locally advanced pancreatic cancer (LAPC). A biopsy of the lesion was only performed, thus revealing the presence of well differentiated adenocarcinoma (Fig. 2).

In order to achieve resectability of this unresectable LAPC, the patient underwent neoadjuvant CCRT for 5 weeks with gemcitabine regimen (gemcitabine $200 \mathrm{mg} / \mathrm{m}^{2}$ intravenously weekly) and total $55 \mathrm{GY}$ of radiation (40 GY in 20 fractions and additional $15 \mathrm{GY}$ in one stereotactic radiosurgery). AP-CT, MRCP and positron emission tomography-computed tomography (PET-CT) were performed one month after neoadjuvant CCRT. This showed a decrease in the size of the pancreatic body mass from about 2.9 to $2 \mathrm{~cm}$. But there was still cancer invasion in the SMV-PV confluence. PET-CT showed hypermetabolic lesion in the
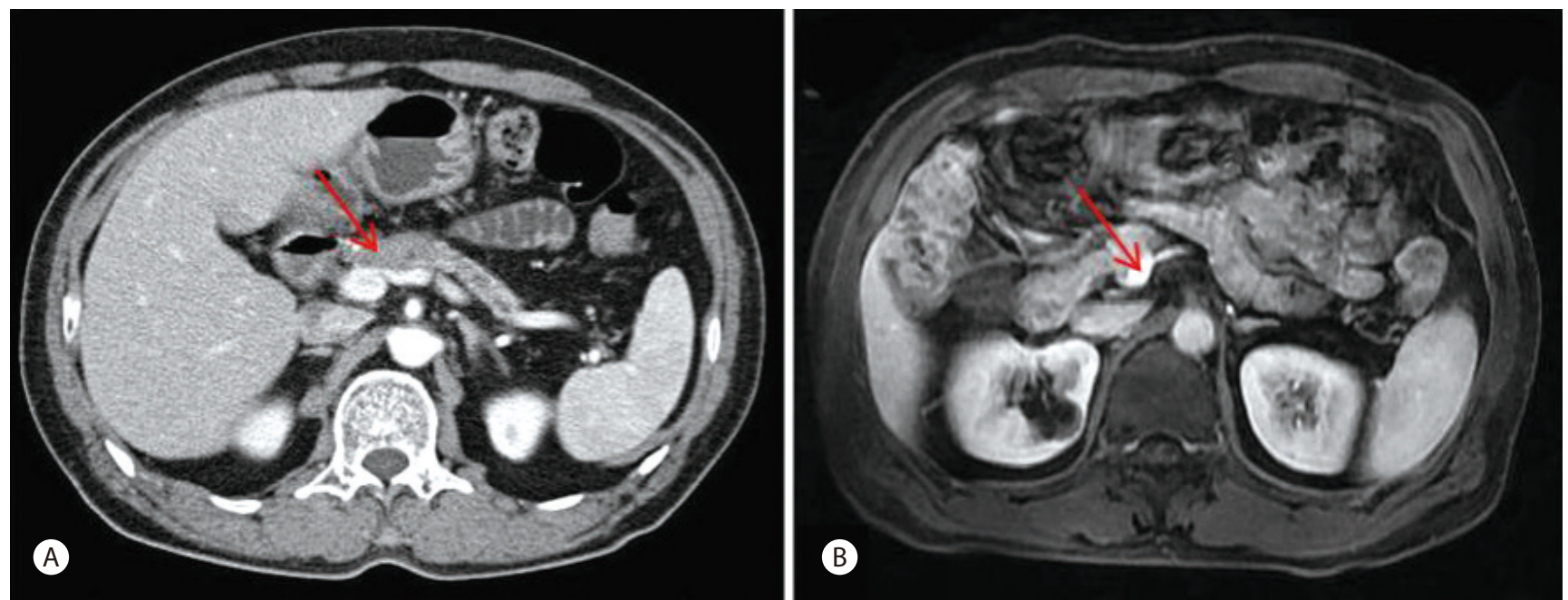

Fig. 1. Figure (A) and (B) present initail images at the time pancreatic cancer was diagnosed. And the arrows indicate pancreatic cancer lesion. (A) Initial abdomino- pelvic computed tomography (AP-CT) showed $2.9 \mathrm{~cm}$ sized mass lesion suspected with pancreatic cancer. (B) Initial magnetic resonance cholangiopancreatography (MRCP) showed invasion of pancreatic cancer in the superior mesenteric vein (SMV) -portal vein (PV) confluence. 


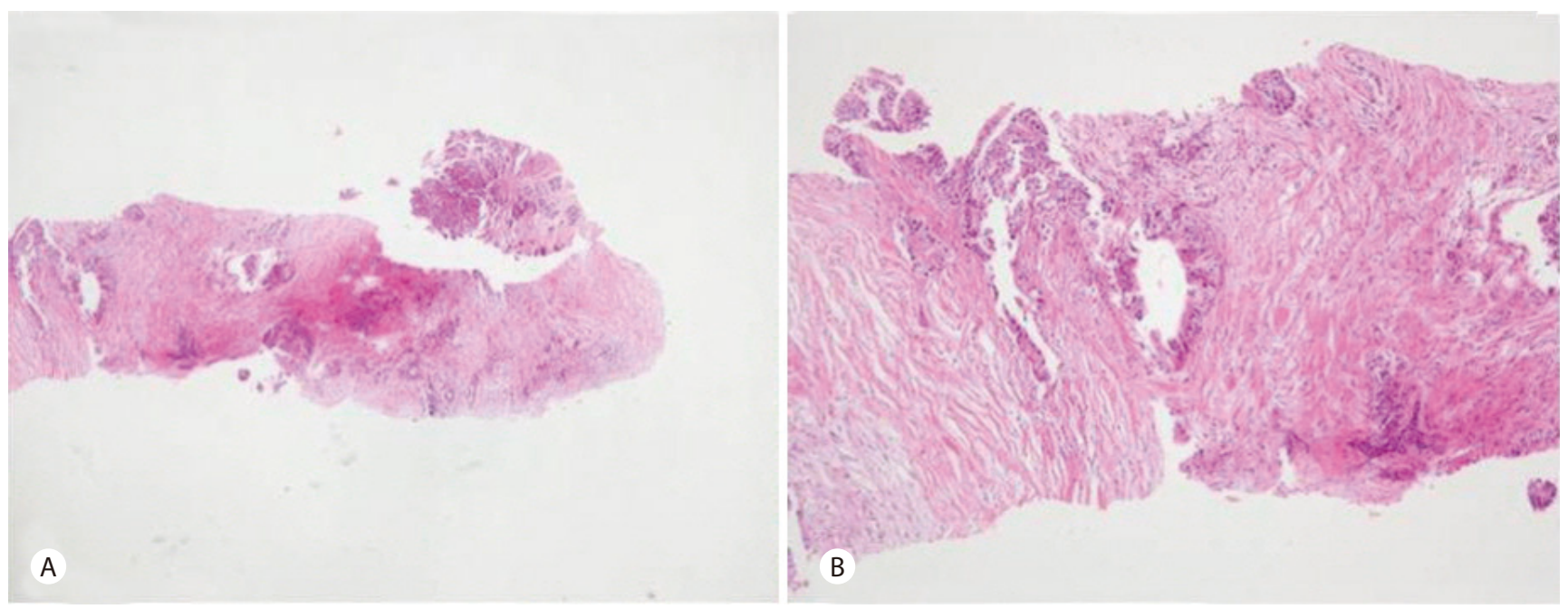

Fig. 2. Biopsy samples of the pancreas were obtained for exploratory laparotomy, showing a histopathological classification of well-differentiated adenocarcinoma with the infiltrative pattern of glands in a desmoplastic stroma. (A: H\&E staining, $\times 40, B$ : H\&E staining, $\times 100$ )

pancreatic body (Fig. 3). Three months after neoadjuvant CCRT, there was a decrease in CA 19-9 levels to $13.88 \mathrm{U} / \mathrm{mL}$. The patient had partial response to neoadjuvant CCRT according to the Response Evaluation Criteria in Solid Tumor (RECIST) version 1.1. But neoadjuvant CCRT for resectability failed due to consistent cancer invasion in major vessels.

The additional gemcitabine chemotherapy with palliative intent was performed. The patient underwent a total of 8 cycles of chemotherapy with gemcitabine regimen (gemcitabine $1000 \mathrm{mg} / \mathrm{m}^{2}$ intravenously on Day $1,8,15$ every 4 weeks) from December 2011 to June 2012. AP-CT after the 2nd cycle of gemcitabine chemotherapy showed a decrease in the size of pancreatic body lesion from 2 to 1.5 cm (Fig. 3). Chemotherapy was continuous for 8 cycles with stable status and there was a constant decrease in CA 19-9 levels from 13.88 to $5.31 \mathrm{U} / \mathrm{mL}$ for gemcitabine chemotherapy.

The patient was generally healthy at the time of diagnosis and not with rapid progression despite unresectable disease. Also, he had gemcitabine chemotherapy with less side effects than other aggressive chemotherapy. He mostly maintained the good performance of Eastern Cooperative Oncology Group (ECOG) performance status 1 during neoadjuvant CCRT and gemcitabine maintenance chemotherapy. And he did not exhibit serious major complications and was not in need of the dose reduction of treat- ment during chemotherapy. But he complained generall weakness after the 8 th cycle of gemcitabine chemotherapy and the gemcitabine chemotherapy was stopped in response to his request due to general weakness.

AP-CT has been followed after gemcitabine chemotherapy. The $1.5 \mathrm{~cm}$ sized pancreatic body lesion had been consistently revealed on AP-CT to about 9 months after gemcitabine chemotherapy. And there had been pancreatic cancer lesion involving and abutting SMV-PV confluence on all AP-CT that had been followed from the time of diagnosis to about 9 months after gemcitabine chemotherapy. So resectability was not acquired during neoadjuvant CCRT and a total of 8 cycles of gemcitabine chemotherapy. But, PET-CT was taken 2 months after gemcitabine chemotherapy and showed no significant increase of fluorodeoxyglucose uptake in the pancreatic cancer lesion which had been present on AP-CT (Fig. 4). On AP-CT that was taken about 13 months after gemcitabine chemotherapy, there was a lack of soft tissue invasion to the pancreatic body involving and abutting SMV-PV confluence. Also, the AP-CT showed markedly atrophied pancreas body. The AP-CT at the time showed complete disappearance of previous primary cancerous lesion (Fig. 4). Based on this CT findings, the patient achieved a complete response. On all CT scans taken after the patient first achieved a complete response, there has been no evidence of pancreatic 

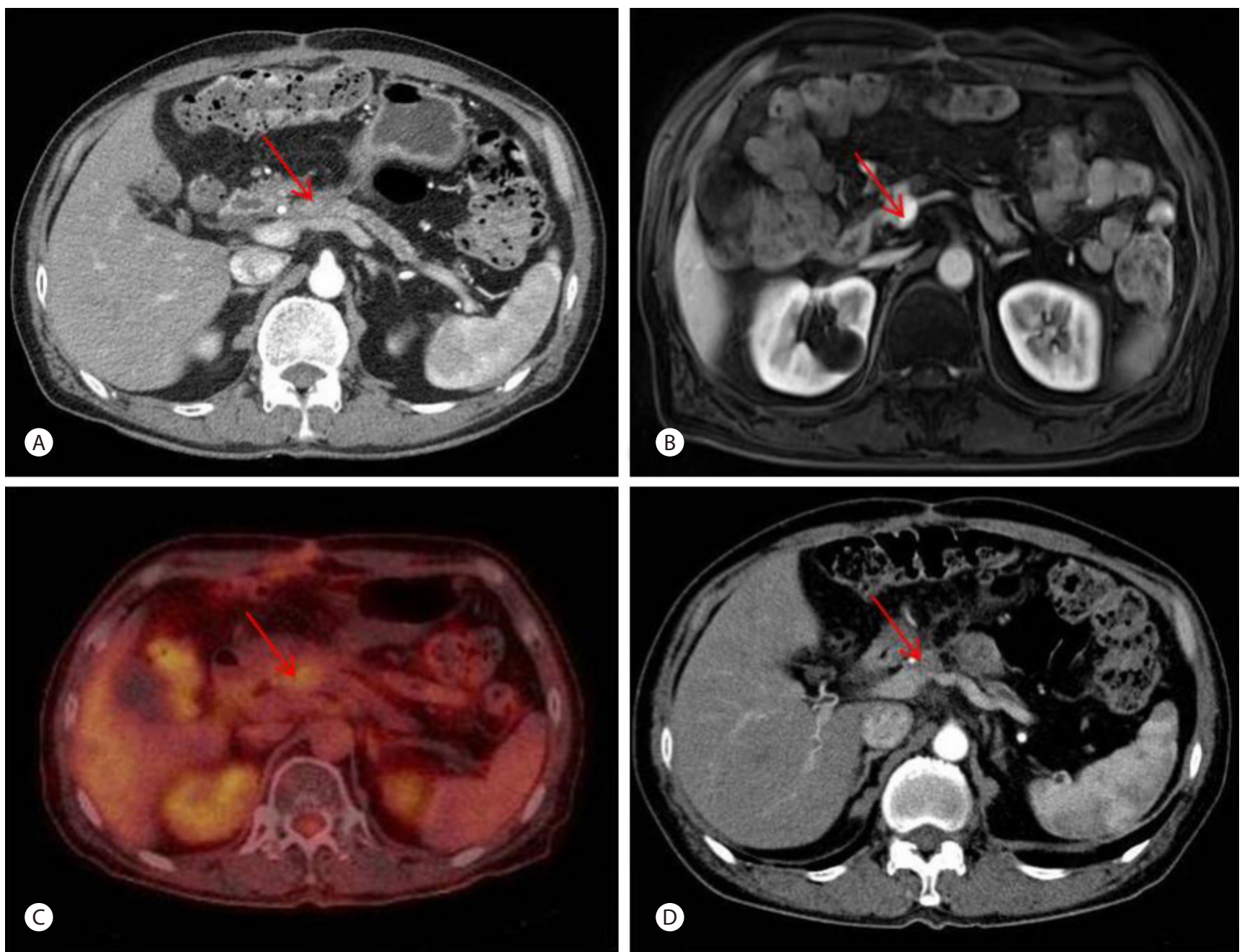

Fig. 3. Figure (A), (B), and (C) present images after neoadjuvant concurrent chmoradiotherapy (CCRT). Figure (D) presents image after the $2 n d$ cycle of gemcitabine chemotherapy. And the arrows indicate pancreatic cancer lesion (A) There was a decrease in the size of pancreastic mass lesion to $2 \mathrm{~cm}$ on abdominopelvic computed tomography (AP-CT). (B) Pancreatic cancer still involved the superior mesenteric vein (SMV) -portal vein (PV) confluence on magnetic resonance cholangio-pancreatography (MRCP) . (C) Positron emission tomography-computed tomography (PET-CT) showed hypermetabolic lesion in the pancreatic body. (D) There was also a decrease in the size of pancreatic mass lesion to $1.5 \mathrm{~cm}$ on AP-CT.

cancer for more than 32 months until the present. And CA 19-9 levels after gemcitabine chemotherapy have been within the normal range without sudden increase (Fig. 5).

\section{DISCUSSION}

Up to date, surgical resection has been recognized as the only curative treatment for pancreatic cancer that is an aggressive disease. ${ }^{3,8}$ It has been reported that there was a significant difference in the overall survival between the group undergoing surgical resection and the group undergoing palliative chemotherapy. ${ }^{6,7}$ A recent meta-analysis indicated that approximately a third of LAPC patients that were initially staged as unresectable achieved resectability after undergoing neoadjuvant treatment, with gaining an overall survival similar to that of patients with initially resectable pancreatic cancer. ${ }^{9}$ On the other hand unresectable LAPC patients who failed in resection after neoadjuvant chemotherapy had almost the same poor prognosis as patients with primary metastatic disease. ${ }^{9}$

Hence, achievement of resectability is the treatment strategy that maximizes the survival rate of pancreatic cancer patients and neoadjuvant chemotherapy has been attempted to achieve resectability in LAPC patients. ${ }^{6}$ In the 

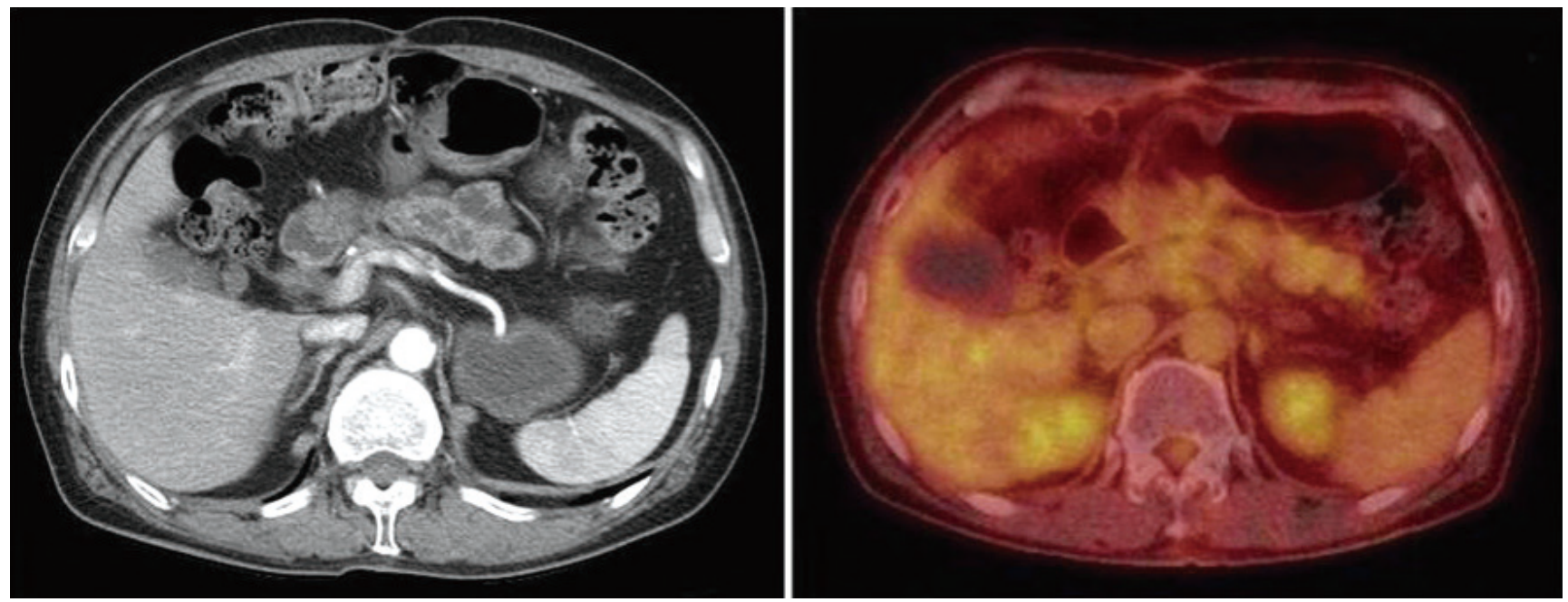

Fig. 4. Figure (A) and (B) present images followed after the completion of gemcitabine chemotherapy. (A) abdominopelvic computed tomography (AP$\mathrm{CT}$ ) followed later showed complete disappearance of previous primary cancerous lesion. (B) There was no significant increase of fluorodeoxyglucose uptake in the pancreatic body on Positron emission tomography-computed tomography (PET-CT) that was taken 2 months after gemcitabine chemotherapy.
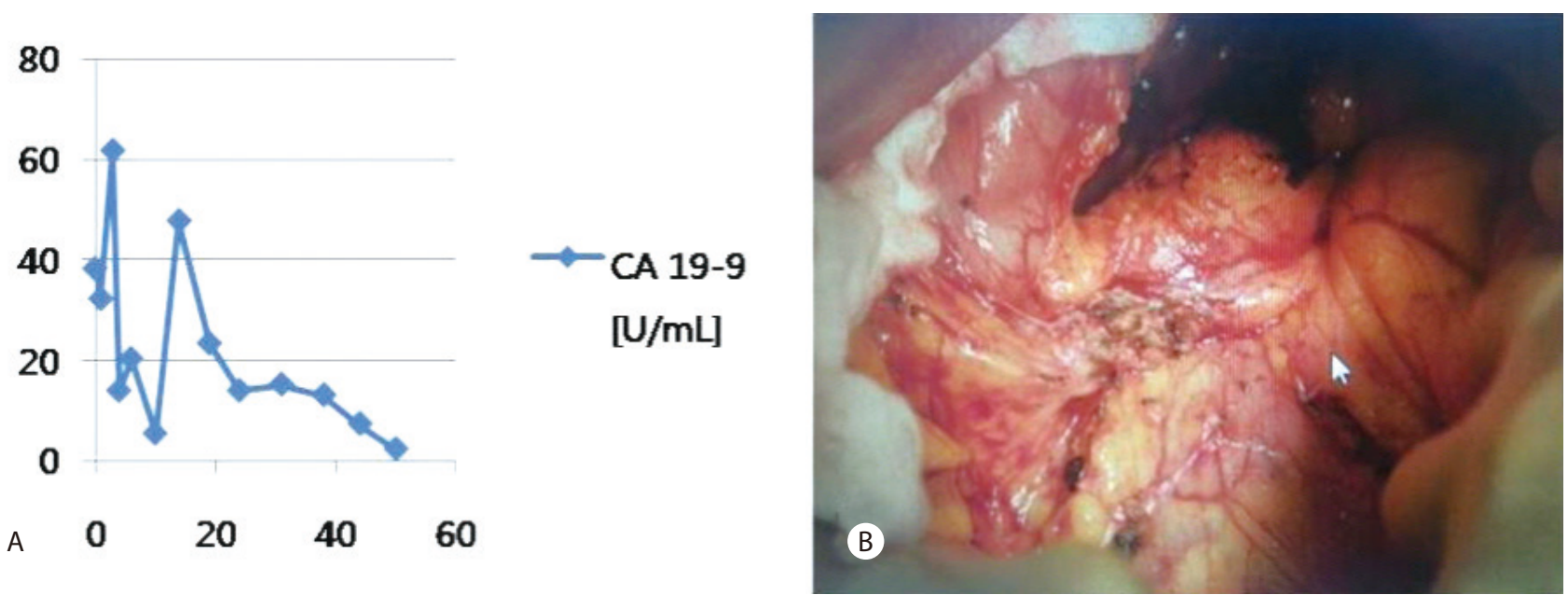

Fig. 5. (A) The blue line presents changes in CA 19-9 levels according to the time. The horizontal axis is the time point (months) of neoadjuvant concurrent chmoradiotherapy (CCRT) for 5 weeks. The vertical axis is the level of CA 19-9. CA 19-9 levels on the whole had decreased during 8 cycles of gemcitabine chemotherapy and have been within normal range without sudden increase thereafter (the normal value of CA 19-9: $<37 \mathrm{U} / \mathrm{mL}$ ). (B) Intraoperative view during exploratory laparotomy. CA, carbohydrate antigen.

cases of failure in resection despite neoadjuvant chemotherapy, a prognosis was very poor as metastatic pancreatic cancer, but there has not been other proper alternative except previous palliative chemotherapy. ${ }^{6}$

In our case, the patient failed in achivement of resectability due to consistent vascular involvement of tumor despite CCRT with gemcitabine regimen. After undergoing a total of 8 cycles of gemcitabine chemotherapy, however, the patient achieved a complete response. This is a very rare case in which a long term complete response has been maintained for more than 32 months on AP-CT scans.

There was one case of other patient with LAPC who underwent 5 cycles of neoadjuvant chemotherapy consisting of gemcitabine plus paclitaxel. On CT scans taken after neoadjuvant chemotherapy, there was unresectability of the pancreatic cancer. Nevertheless, the patient underwent surgical exploration. In contast with CT findings, operative 
findings for exploration indicated resectability of pancreatic cancer and the patient achieved a complete resection with a pathologic complete response. ${ }^{6}$

This could be also applied to our case. The patient in our case was strongly suspected with LAPC which involved major vessels on $\mathrm{CT}$ findings. The resectability in our patient depended whether cancer invasion in SMV-PV confluence on AP-CT actually existed. So surgical exploration was needed to evaluate the chance of resectability in spite of unresectability on imaging study. Although pathologic confirmation of pancreatic cancer was not performed before surgical exploration, the possibility of pancreatic cancer was also very high on images. Therefore, surgical exploration was performed for pathologic confirmation and evalation of resectability with the patient's request.

There was the other case. In this case, a patient with advanced pancreatic head cancer had a complete response by imaging and relapse-free survival for 11 years after gemcitabine chemotherapy. The patient complained of abdominal discomfort 11 years after a complete response and then underwent CT scans. Despite a lack of evidence of pancreatic cancer on CT scans, adenocarcinoma was identified on brushing cytology during endoscopic retrograde cholangiography (ERCP). So, he subsequently presented with local tumor recurrence and underwent pancreaticoduodenectomy followed by gemcitaine plus S-1 chemotherapy. ${ }^{10}$

Considering two previous cases, a complete response by imaging in pancreatic cancer may not mean a pathological complete response. Surgical exploration can be occasionally needed for pancreatic cancer patients with a complete response by imaging after chemoradiotherapy. Perhaps, physicians will consider surgical exploration if the possibility of a pathologic complete response is very high in patients who do not achieve a complete response on images or disease recurrence is strongly suspected in patients with a complete response by imaging. Although surgical exploration is required for the confirmation of a complete response by physicians, the evaluation of a complete response might be depended on image studies in cases that the operative risk of a patient is high or he does not want surgical exploration.

The primary cancerous lesion of our patient had been under stable status long before he achieved a complete response by imaging. The evidence of malignancy was not found on PET-CT followed at the time. And CA 19-9 levels on the whole had decreased during 8 cycles of gemcitabine chemotherapy and have been within normal range without sudden increase thereafter. And our patient did not have complained clinically meaningful symptoms during and after 8 cycles of gemcitabine chemotherapy. So he was with low risk for cancer recurrence at the time that a complete response by imaging was achieved. He already underwent a surgical exploration before neoadjuvant chemotherapy. In the case of additional surgery, there was a high possibility that the operation would be difficult due to adhesion. There was also operative risk in that he was old age and had arrhythmia before. Above all, he did not want additional surgery. Therefore, we did not perform additional surgical exploration considering the above reasons after a complete response by imaging.

Our case has some significance. First, our patient achieved a complete response solely with additional chemotherapy despite of unresectability in pancreatic cancer that complete resection is curative therapy. So, this contributed to the concept establishment of chemotherapy as not palliative but curative treatment. Second, pancreatic cancer patients have poor prognosis and frequent recurrence. However, our patient has maintained a long term complete response without disease recurrence.

\section{요 약}

저자들은 절제 불가능한 국소진행형 췌장암 환자가 동시 적 항암화학방사선요법 및 gemcitabine 유지 항암화학요법 을 받은 후 장기간 동안 완전 관해를 유지하고 있는 증례를 보고하는 바이다. 이 증례를 통해 다른 절제 불가능한 국소 진행형 췌장암 환자들에게도 동시적 항암화학방사선요법 및 gemcitabine 유지 항암화학요법 치료를 시도해 볼 수 있을 것이다. 
국문 색인: 췌장암, 항암화학방사선요법, 젬시타빈, 항암화 학요법

\section{Conflicts of Interest}

The author has no conflicts to disclose.

\section{REFERENCES}

1. Boone BA, Steve J, Krasinskas AM, et al. Outcomes with FOLFIRINOX for borderline resectable and locally unresectable pancreatic cancer. J Surg Oncol 2013;108:236-241.

2. Reynolds RB, Folloder J. Clinical management of pancreatic cancer. J Adv Pract Oncol 2014;5:356-364.

3. Turner K, Levi Sandri GB, Boucher E, et al. Complete radiological response of an initially locally advanced unresectable pancreatic cancer to chemoradiotherapy using FOLFIRINOX regimen: report of a case. Clin Res Hepatol Gastroenterol 2015;39:e29-e31.

4. Tinkl D, Grabenbauer GG, Golcher $H$, et al. Downstaging of pancreatic carcinoma after neoadjuvant chemoradiation. Strahlenther Onkol
2009:185:557-566.

5. Neoptolemos JP, Stocken DD, Friess $H$, et al. A randomized trial of chemoradiotherapy and chemotherapy after resection of pancreatic cancer. N Engl J Med 2004;350:1200-1210.

6. Hartlapp I, Müller J, Kenn W, et al. Complete pathological remission of locally advanced, unresectable pancreatic cancer (LAPC) after intensified neoadjuvant chemotherapy. Onkologie 2013;36:123-125.

7. Vincent A, Herman J, Schulick R, Hruban RH, Goggins M. Pancreatic cancer. Lancet 2011;378:607-620.

8. Valeri S, Borzomati D, Nappo G, Perrone G, Santini D, Coppola R. Complete pathological response after FOLFIRINOX for locally advanced pancreatic cancer. The beginning of a new era? Case report and review of the literature. Pancreatology 2014;14:425-430.

9. Gillen S, Schuster T, Meyer Zum Büschenfelde C, Friess H, Kleeff J. Preoperative/neoadjuvant therapy in pancreatic cancer: a systematic review and meta-analysis of response and resection percentages. PLoS Med 2010;7:e1000267.

10. Uchihara T, Yamashita $Y$, Hualin W, et al. Recurrence 11 years after complete response to gemcitabine, 5-Fluorouracil, and Cisplatin chemotherapy followed by radiotherapy in a patient with advanced pancreatic cancer: a case report. Anticancer Res 2015;35:2867-2871. 